\title{
INDICADORES DA PRODUÇÃo COLABORATIVA NA ARQUITETURA DA INFORMAÇÃO
}

\author{
COLLABORATIVE PRODUCTION INDICATORS IN INFORMATION \\ ARCHITECTURE
}

INDICADORES DE PRODUCCIÓN DE COLABORACIÓN EN LA CONFIGURACIÓN DE LA INFORMACIÓN

${ }^{1}$ Zayr Claudio Gomes da Silva, ${ }^{2}$ Edivanio Duarte de Souza

${ }^{1}$ Universidade Federal da Paraíba, ${ }^{2}$ Universidade Federal de Alagoas

\section{Correspondência}

1Zayr Claudio Gomes da Silva

João Pessoa,PB

Email: zayr10@gmail.com

ORCID: http://orcid.org/0000-0002-2758-

$\underline{3424}$

Submetido em: 24-10-2017

Aceito em: 21-03-2017

Publicado: 22-03-2017

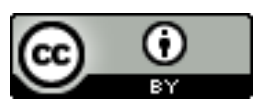

JITA: KE. Architecture. 
RESUMO: A arquitetura da informação é considerada como um domínio estratégico de produção colaborativa da Ciência da Informação. Descrevem-se as condições da produção colaborativa na arquitetura da informação, considerando-a como subárea de estudo da Ciência a Informação. Para tanto, abordam-se, especificamente, indicadores da produção científica que compreendem temáticas de estudo, tipologia e formação da autoria, programas de pós-graduação e áreas a que se encontra vinculada, entre outros. Trata-se de uma pesquisa exploratória e descritiva. Mapeia-se a produção científica do Encontro Nacional de Pesquisa em Ciência da Informação (ENANCIB), no período de 2003 a 2013, no repositório “Questões em Rede". Utiliza-se a bibliometria para identificar elementos paratextuais e textuais que formam indícios da produção colaborativa na arquitetura da informação. Constatou-se a pluralidade na formação acadêmica dos pesquisadores que abordam a arquitetura da informação, o compartilhamento de linguagens, alguns indícios das convergências disciplinares a partir da colaboração em coautoria, bem como um plexo de relações através das citações indiretas que representam o compartilhamento de elementos teórico-metodológicos na produção interdisciplinar. Além disso, a formação acadêmica dos pesquisadores com maior índice de produtividade é vinculada principalmente à Biblioteconomia e à Ciência da Computação. Considera-se que a produção colaborativa na arquitetura da informação se apresenta como processo de produção multidisciplinar, constituindo-se em um domínio convergente que possibilita a efetividade de práticas interdisciplinares na Ciência da Informação.

PALAVRAS-CHAVE: Ciência da Informação. Epistemologia da Ciência da Informação. Produção Colaborativa na Arquitetura da Informação. Produção Colaborativa na Ciência da Informação.

ABSTRACT: Information architecture is considered as a strategic area of collaborative production of information science. It describes the conditions of collaborative production in information architecture, considering it as subarea study of the Information Science. Therefore, addresses specifically indicators of scientific production comprising thematic study, type and formation of authorship, graduate programs and areas to which it is bound, etc. This is an exploratory and descriptive research. Maps to scientific production of the National Meeting of Research in Information Science (ENANCIB) in the 2003-2013 period in the repository "Network Issues". It is used bibliometrics to identify paratextual and textual elements that form evidence of collaborative production in information architecture. It found the plurality in academic researchers to address the information architecture, languages sharing, some evidence of disciplinary convergences from the collaboration in co-authorship, as well as a plexus of relations through indirect quotations representing elements sharing theoretical and methodological in interdisciplinary production. In addition, academic researchers with higher productivity index is linked mainly to the Library and Computer Science. It is considered that the collaborative production in the information architecture presents itself as a multidisciplinary production process, thus becoming a converged domain that enables the effectiveness of interdisciplinary practices in Information Science.

KEYWORDS: Information Science. Epistemology of Information Science. Collaborative Production in Information Architecture. Collaborative Production in Information Science.

RESUMEN: Arquitectura de la información es considerada como un área estratégica de la producción colaborativa de ciencias de la información. las condiciones de producción colaborativa se describen en la arquitectura de la información, teniendo en cuenta que como el estudio de la subzona de la Ciencia de la Información. Por lo tanto, para abordar específicamente los indicadores de producción científica que comprenden estudio temático, el tipo y la formación de la autoría, los programas de postgrado y las áreas a la que está vinculada, entre otros. Se trata de una investigación exploratoria y descriptiva. Mapas a la producción científica de la Reunión Nacional de Investigación en Ciencias de la Información (Enancib) en el período 2003-2013, el repositorio "Cuestiones de red". Se utiliza la bibliometría para identificar los elementos paratextuales y textuales que forman pruebas de producción colaborativa en la arquitectura de la información. Se encontró que la pluralidad de los investigadores académicos para hacer frente a la arquitectura de la información, idiomas compartir, alguna evidencia de convergencias disciplinarias de la colaboración con otros autores, así como un plexo de relaciones a través de las citas indirectas que representan los elementos que comparten teórico y metodológico en la producción interdisciplinaria. Además, los investigadores académicos con mayor índice de productividad está ligada principalmente a la Biblioteca y Ciencias de la Computación. Se considera que la producción colaborativa en la arquitectura de la información se presenta como un proceso de producción multidisciplinar, convirtiéndose así en un dominio convergente que permite a la eficacia de las prácticas interdisciplinares en Ciencias de la Información.

PAlABRAS ClaVE: Ciencias de la Información. Epistemología de la Ciencias Información. Producción de colaboración en la Arquitectura de la Información. Producción de colaboración en Ciencias de la Información.

\begin{tabular}{l|c|c|c|c|c}
\hline (C) RDBCI: Rev. Digit. Bibliotecon. Cienc. Inf. & Campinas, SP & v.15 & n.2 & p. 368-388 & maio/ago. 2017 \\
\hline
\end{tabular}




\section{INTRODUÇÃ̃}

$\mathrm{Na}$ contemporaneidade, as práticas de integração do conhecimento, a multidisciplinaridade, a interdisciplinaridade e a trandisciplinaridade, ganham status de movimentos de produção colaborativa do conhecimento cientifico, na medida em que reivindicam essencialmente a complexidade da natureza, buscando a integração dos objetos, que se apresentam cada vez mais fragmentados, em decorrência da crescente especialização do saber. De modo mais preciso, essas buscam a superação do estado de fragmentação promovido pelo modelo de analiticidade característico da ciência moderna. Esses movimentos resultam de relações cooperativas entre os diferentes atores envolvidos na produção científica, desde pesquisadores, disciplinas teorias e métodos, entre outros.

A produção científica, nesse contexto, se caracteriza como colaborativa, na medida em que ela é constitutiva daqueles movimentos, que vem se mostrando como um conjunto de alternativas factível para o alcance da complexidade dos objetos por intermédio de abordagens disciplinares integrativas, considerando a multidimensionalidade desses objetos e das relações passíveis de serem estabelecidas entre temáticas, conceitos, perspectiva, teorias, linguagens e metodologias, entre outros, nas diversas formas de saber.

A Ciência da Informação, campo de conhecimento científico dedicado ao estudo do fenômeno informacional e de processos, tecnologias e metodologias que o envolvem, é caracterizada por uma multiplicidade de fundamentos epistemológicos que condicionam as práticas colaborativas. Nesse contexto, podem-se destacar algumas relações históricas com diferentes áreas de conhecimento, tais como a Biblioteconomia de Jesse Shera (1903-1982), a Informação Científica de Mikhailov (1905-1988), a Documentação de Paul Otlet (1868-1944), a Teoria Matemática da Comunicação de Shannon (1916-2001) e a Engenharia e Recuperação da Informação de Vannevar Bush (1890-1974).

Historicamente, a produção colaborativa emerge como um dos principais fundamentos epistemológicos na Ciência da Informação, condicionada pela complexidade do objeto informacional, pela formação plural de seus pesquisadores e pela consequente convergência disciplinar com áreas com as quais dialoga (SOUZA, 2011).

Nesse campo híbrido de estudo, merece destaque a temática "arquitetura da informação", que é um domínio de estudo convergente, na medida em que pode ser considerado um espaço epistêmico estratégico para estudos colaborativos dedicados à produção interdisciplinar, tendo em vista alguns relacionamentos teórico-metodológicos de diferentes áreas, como a Biblioteconomia (organização e classificação de conteúdo) e a Ciência da Computação (apresentação de conteúdo na Web, sistemas de navegação e sistemas de recuperação da informação), que abordam processos, fluxos e necessidades informacionais.

Assim, o presente artigo busca descrever as condições da produção colaborativa no

\begin{tabular}{c|c|c|c|c|c}
\hline (C) RDBCI: Rev. Digit. Bibliotecon. Cienc. Inf. & Campinas, SP & v.15 & n.2 & p. 368-388 & maio/ago. 2017 \\
\hline
\end{tabular}


domínio arquitetura da informação, considerando-a como subárea de estudo da Ciência a Informação. Para tanto, aborda, especificamente, indicadores da produção científica que compreendem temáticas de estudo, tipologia e formação da autoria, programas de pósgraduação e áreas a que se encontra vinculada, entre outros.

É importante considerar que se trata de parte dos resultados da pesquisa de dissertação "A produção interdisciplinar na Ciência da Informação: abordagem nos domínios da arquitetura da informação", defendida junto ao Programa de Pós-Graduação da Ciência da Informação da Universidade Federal da Paraíba (PPGCI/UFPB), que teve como objetivo compreender os elementos teórico-metodológicos que contribuem com a produção colaborativa nos domínios da arquitetura da informação na Ciência da Informação.

\section{2 fundamentos da PROdUÇÃo colaborativa NA CIÊNCIA DA INFORMAÇÃO}

A produção colaborativa do conhecimento científico se configura a partir de uma perspectiva teórico-metodológica que visa à complexidade dos objetos/fenômenos, às relações disciplinares, aos diálogos com as mais variadas formas de saber e às conexões externas da ciência, considerando os aspectos sociopolíticos e econômicos relacionados à atividade científica.

Conforme Japiassu (1976) e Pombo (2008), três movimentos de integração do saber ganham destaque na produção de conhecimento científico, a multidisciplinaridade, a interdisciplinaridade e a transdisciplinaridade. Estes se aproximam e se entrelaçam conceitualmente, entretanto, segundo Pombo (2008), podem ser compreendidos como um continuum, de modo que a integração do conhecimento se eleva do gênero da coordenação paralela (multidisciplinaridade e pluridisciplinaridade), passando por uma combinação de conhecimento em convergência (interdisciplinaridade), até uma perspectiva de fusão holística (transdisciplinaridade).

Nesse contexto, conforme Souza (2015), essas práticas integrativas ganham hodiernamente o status de produção colaborativa já que, em sua dinâmica, compreende um complexo de relações multidisciplinares, interdisciplinares e transdisciplinares que potencializam o compartilhamento de conceitos, métodos e teorias entre diferentes áreas do conhecimento. O fato é que essa dinâmica de colaboração ultrapassa os enfoques centrados em fronteiras e limites epistemológicos, na medida em que o processo colaborativo se redimensiona a partir das associações entre diversos atores, notadamente, pesquisadores, conceitos, metodologias, teorias e, em maior ou menor medida, disciplinas.

A inteligência coletiva, nesta perspectiva, se destaca como um dos elementos característicos da produção colaborativa, considerando que "aquilo que o indivíduo não pode isoladamente, a soma dos indivíduos, reunidos numa coletividade poderá, [...] reunidos

\begin{tabular}{|c|c|c|c|c|c|}
\hline (C) RDBCI: Rev. Digit. Bibliotecon. Cienc. Inf. & Campinas, SP & $\mathrm{v} .15$ & n.2 & p. $368-388$ & maio/ago. 2017 \\
\hline
\end{tabular}


cooperativa e coletivamente" (DOMINGUES, 2005, p. 29). Esta propriedade em conjunto forma base para uma produção colaborativa, tendo em vista que problemas e objetos complexos necessitam de múltiplos olhares, de modo que possam fornecer soluções a partir de diferentes ideias, conceitos, perspectivas, abordagens, teorias e metodologias, entre outros.

Assim, a inteligência coletiva, tratada como um pressuposto básico da produção colaborativa se configura a partir da participação de diversos sujeitos, habilidades e competências, e, complementarmente, através de discursos disciplinares baseados em conceitos e métodos de diferentes áreas. No que se relaciona particularmente à interdisciplinaridade, que se apresenta como um dos movimentos de produção colaborativa, tem-se como principais fundamentos a complexidade dos objetos e a relação entre disciplinas. A rigor, segundo Japiassu (1976), ela se efetiva por intermédio da integração de conceitos, teorias, métodos e linguagens entre diferentes disciplinas.

$\mathrm{Na}$ Ciência da Informação, a produção colaborativa vem se caracterizando pela variedade dos fundamentos, desde suas demarcações históricas às relações interdisciplinares a partir de diferentes conceitos, teorias e disciplinas. Com efeito, a interdisciplinaridade é discutida como um dos fundamentos da Ciência da Informação, que se caracteriza em torno de três variáveis, a saber, a complexidade da informação, a formação plural dos pesquisadores e a consequente convergência entre as disciplinas que fazem interface com a área (GONZÁLEZ DE GÓMEZ, 2001; PINHEIRO, 1997; SOUZA, 2011).

A complexidade do objeto informação se evidencia, principalmente, por meio dos aspectos conceituais, tendo em vista a multiplicidade conceitual coexistente em diferentes noções e sentidos, discutidos em várias disciplinas e áreas do conhecimento. Capurro e Hjørland (2007, p. 160) afirmam que o conceito de informação se constitui colaborativamente, pois "[várias disciplinas científicas] usa[m] o conceito de informação dentro de seu próprio contexto e com relação a fenômenos específicos".

A interdisciplinaridade na Ciência da Informação em relação ao objeto informação se configura a partir da "traduzibilidade de conceitos" discutida por Domingues (2005), no contexto da produção colaborativa. Em uma perspectiva mais ampla, o autor observa que:

a abordagem transdisciplinar não trata de estabelecer vários discursos disciplinares isolados sobre um mesmo objeto - multidisciplinaridade ou reunir os vários saberes e discursos dentro de um único discurso globalizante - interdisciplinaridade. Ela põe em contato as várias disciplinas e saberes específicos até eles compenetrarem-se, transfigurarem-se e formarem um novo campo, tal como aconteceu ao se estudar a energia desde meados do século passado e, recentemente, ao se estudar as cidades e seus problemas como transporte, violência, espaço físico e educação [e, por que não a informação?]. Nesse contato, as disciplinas se transformam e os conceitos transmutam, por destruição e reconstrução constantes e pela contínua migração de um campo para o outro, como do pensamento ao visível e vice-versa, não mais se preservando inalteradas as formas e as fronteiras demarcatórias anteriores

\begin{tabular}{l|l|l|l|l|l}
\hline (C) RDBCI: Rev. Digit. Bibliotecon. Cienc. Inf. & Campinas, SP & v.15 & n.2 & p. 368-388 & maio/ago. 2017 \\
\hline
\end{tabular}


(DOMINGUES, 2005, p. 44).

Além disso, é importante considerar que a Ciência da Informação emerge a partir da explosão tecnocientífica e de questões referentes ao acesso e à recuperação da informação e do conhecimento, que envidam esforços multidisciplinares. Saracevic (1996) afirma que os problemas tratados pela área requerem a contribuição de pesquisadores de diferentes disciplinas, dentre os quais se podem citar engenheiros, químicos, filósofos, linguistas, bibliotecários, matemáticos e cientistas da computação. Desse modo, as justaposições disciplinares como base da multidisciplinaridade se formam na Ciência da Informação, tornando-se condição da produção interdisciplinar na área.

A produção interdisciplinar da Ciência da Informação é realizada a partir de crescentes relações com diversas disciplinas, tendo em vista a necessidade de convergências de diferentes áreas para resolução dos problemas informacionais. Com efeito, Souza (2011, p. 159) compreende que, no desenvolvimento da do campo epistemológico, "há uma ampliação com a inserção de novos campos de conhecimento por intermédio de novos pesquisadores e da inclusão de novas temáticas no domínio de estudo".

Não obstante os esforços realizados por pesquisadores de diferentes áreas do conhecimento, de acordo com Saracevic (1999), há na Ciência da Informação dificuldade de integração de duas vertentes, uma orientada ao usuário e ao conteúdo, influenciada pela Biblioteconomia, e outra orientada à tecnologia, relacionada à Ciência da Computação.

É justamente neste espaço tanto conflituoso quanto epistêmico que a arquitetura da informação emerge como temática de estudo na Ciência da Informação, que possibilita movimentos de integração em torno da produção colaborativa, na medida em que aborda, a um só tempo, a estruturação de conteúdos em ambientes digitais, de forte base tecnológica, e aos usuários, visando às necessidades informacionais destes. De modo mais preciso, trata-se de um domínio de estudo que se dedica a reflexões sobre os processos de organização e recuperação da informação, integrando conteúdos, tecnologias e usuários.

\section{ARQUITETURA DA INFORMAÇÃO: UM DOMÍNNIO CONVERGENTE NA CIÊNCIA DA INFORMAÇÃO}

A arquitetura da informação surge no cenário de explosão de dados e informação, momento em que muito se tinha e pouco se compreendia. Wurman (1997) cunhou o termo information architecture como a arte de criar instruções para organizar um espaço, tentando tornar o complexo simples, considerando que a quantidade de informação resultaria na dificuldade de compreensão, em decorrência da crescente "ansiedade da informação". Assim, se reiterava uma adequada ordenação dos dados e informação que se encontravam de forma desordenada. 
De acordo com Morville e Rosenfeld (2006), a arquitetura da informação passa a ser discutida principalmente tendo em vista o contexto digital que se caracteriza pelo avanço exponencial das tecnologias da informação e comunicação, principalmente a Internet, e pelo consequente redimensionamento da explosão da informação.

Nesse contexto, Agner (2009, p. 89) afirma que a arquitetura da informação pode ser definida como uma "metadisciplina preocupada com o projeto, a implementação e manutenção de espaços informacionais digitais para o acesso humano, a navegação e o uso [...]”. Complementarmente, Morville e Rosenfeld (2006) entendem-na como um projeto de elementos visuais e informacionais, com enfoque na organização (classificação e ordenamento dos conteúdos), na navegação (orientação aos usuários em páginas e hiperlinks) na rotulação ou rotulagem (representação de rótulos linguísticos como palavras e conceitos) e na busca (procura e recuperação dos conteúdos em ambientes digitais) de conteúdos.

Numa maior aproximação às questões da produção colaborativa, é importante considerar que, para Santos e Vidotti (2009), a partir da estrutura de informações disponíveis nos ambientes informacionais digitais em termos de tecnologias da informação e comunicação, podem-se "desenvolver arquiteturas informacionais e computacionais [...]", de modo que se possa enfatizar "a interdisciplinaridade e transdisciplinaridade, com uma verticalidade investigativa realizada no diálogo com os diversos campos do conhecimento e centrada no humano" (SANTOS; VIDOTTI, 2009).

Para além, a arquitetura da informação, em perspectiva multidisciplinar, emerge dentro de uma pluralidade de elementos interconceituais delimitados a partir de relações com outras temáticas, disciplinas e áreas do conhecimento. Autores como Macedo (2005) e Morville e Rosenfeld (2006) discutiram brevemente as relações da arquitetura da informação com outras disciplinas e temáticas, observando que a temática converge com temáticas/disciplinas, tais como o Design Gráfico, nos aspectos visuais, o Design de Interação, nas atividades relacionadas aos softwares e às interfaces de sistemas de informação, a Comunicação e o Jornalismo, na disseminação da informação em notícias e edição de conteúdos na Web, e a Ciência da Computação, nas técnicas de banco de dados, no armazenamento de conteúdos e nas linguagens de programação para websites.

Além disso, consideram que a arquitetura da informação se relaciona com a Biblioteconomia e Ciência da Informação (Library and Information Science), a partir da organização da informação e do seu acesso em sistemas de informação, considerando que os profissionais dessas áreas são treinados para trabalhar com tecnologias de busca, navegação e indexação (MORVILLE; ROSENFELD, 2006).

A arquitetura da informação vem se revelando como um domínio estratégico para a realização de estudos e a consolidação de práticas colaborativas no campo da Ciência da Informação, porém, não se pode perder de vista que: 
este "diálogo entre disciplinas" seja extremamente positivo para a formação e o desenvolvimento da disciplina. Entretanto, ao se estabelecer de forma interdisciplinar no âmbito da pós-modernidade, a disciplina precisa delinear sua própria história, e fundamentar-se em bases teóricas consistentes, para que possa identificar seus limites e compreender de que forma se relaciona com outras áreas do conhecimento. 'Ressalta-se aqui a importância de adaptar as teorias e modelos importados de outras áreas, e não somente importá-los. Há que se considerar as particularidades da disciplina para que seja possível adequar os conhecimentos absorvidos de outras áreas' (MACEDO, 2005, p. 158-159, grifo nosso).

Esta ponderação de Macedo (2005) se aproxima da postura crítica de Mostafa (2005), na medida em que esta considera que "não se deve temer as relações" interdisciplinares, desde que estas possam se estabelecer a partir de mudanças, adaptações e diferenciações fundamentadas teórica e metodologicamente, buscando novos conceitos, conhecimentos e outras novas relações.

A arquitetura da informação pode ampliar e se consolidar no campo da Ciência da Informação como domínio convergente, considerando as possíveis relações interdisciplinares em torno do objeto informacional e dos processos de organização e recuperação da informação que o envolvem.

\section{PROCEDIMENTOS METEDOLógicos}

Trata-se de uma pesquisa exploratória e descritiva, que teve como universo as comunicações realizadas no Encontro Nacional de Pesquisa em Ciência da Informação (ENANCIB), no período de 2003 a 2013. Nesse recorte, considerou-se que, a partir dos anos 2000, foi estabelecido o Grupo de Trabalho da Associação Nacional de Pesquisa e PósGraduação em Ciência da Informação (GT/ANCIB) ${ }^{1}$ dedicado aos estudos históricos e epistemológicos da área, possibilitando as discussões sobre a natureza da informação em suas bases teórico-conceituais e epistemológicas. Além disso, esta delimitação teve como referência a constatação do período de crescimento exponencial da produção científica sobre informação e tecnologia no contexto da arquitetura da informação, no maior evento da área.

$\mathrm{Na}$ fase exploratória, que corresponde à centralidade deste trabalho, buscou-se aproximar dos elementos envolvidos na pesquisa, como o objeto, as temáticas e o contexto, tendo em vista o escopo e abrangência do estudo. Para tanto, mapeou-se a produção científica

\footnotetext{
${ }^{1}$ É importante esclarecer, conforme Lara e Smit (2010), que, no início da realização do ENANCIB, o GT 1 foi dedicado à temática informação tecnológica (com variações ao longo dos primeiros anos) e o GT 8 aos estudos históricos da Ciência da Informação. Porém, desde 2005, o GT 1 passou a ser denominado de Estudos Históricos e Epistemológicos, sendo excluído naquele momento o GT responsável pelos estudos sobre tecnologia. Entretanto, a partir de 2008, o GT 8 passou a ser denominado de Informação e Tecnologia, mantendo-se até a configuração atual.
}

\begin{tabular}{l|c|c|c|c|c}
\hline (C) RDBCI: Rev. Digit. Bibliotecon. Cienc. Inf. & Campinas, SP & v.15 & n.2 & p. 368-388 & maio/ago. 2017 \\
\hline
\end{tabular}


no repositório "Questões em Rede", na coleção BENANCIB, que indexa a produção científica dos ENANCIBs.

O mapeamento foi realizado, no dia 20 de novembro de 2015, no catálogo do repositório, "navegando por palavras-chave" - assunto. A partir do mapeamento da produção, foram coletadas 32 (trinta e duas) comunicações sobre a temática arquitetura da informação, considerando 7 palavras-chave classificadas naquele catálogo, a saber, arquiteto da informação (1), arquitetura da informação (25), arquitetura de informação (2), arquitetura da informação para web (1), arquitetura da informação pervasiva (1), arquitetura da informação - sistemas de organização do conhecimento (1) e arquitetura de metadados (1). Para confirmação da temática, fora observado a abordagem efetiva da temática, partir da leitura dos resumos ${ }^{2}$. Os dados coletados nesta fase foram nomes de autores, formações acadêmicas (graduação, mestrado e doutorado), Grupo de Trabalho (GT), programa de pós-graduação e instituição a que a produção se encontra vinculada.

Posteriormente, na fase descritiva, buscou-se identificar e caracterizar os indícios da produção colaborativa na Ciência da Informação nos domínios da arquitetura da informação, por meio de indicadores bibliométricos. Com efeito, a bibliometria é tratada como o "estudo dos aspectos quantitativos da produção, disseminação e uso da informação registrada" (MACIAS-CHAPULA, 1998, p. 134). Desse modo, as análises bibliométricas possibilitaram explanar os indicadores que indiciam as bases da produção interdisciplinar da Ciência da Informação nos domínios da arquitetura da informação.

No entanto, sabe-se da limitação dos procedimentos com base na bibliometria no que tange ao seu enfoque quantitativo de análise de dados. Logo, seguindo os esclarecimentos de Alvarenga (1998) de que o "potencial gerado pela bibliometria se apresenta como insumos valiosos para o desenvolvimento de estudos arqueológicos e epistemológicos regionais, ou seja, dos campos específicos do saber", utilizam-se de algumas noções de formação discursiva, que, segundo Foucault (2008), correspondem ao conjunto de condições que, a um só tempo, define o domínio do campo discursivo e as condições mais amplas do processo discursivo.

Para Foucault (2008), as formações discursivas de um campo/positividade são delineadas a partir de um regime de existência, tomando como base três elementos: a) as demarcações das superfícies primeiras de emergência, que possibilitam a descrição de um domínio discursivo, dando-lhe condições básicas de um objeto do campo; b) as descrições das instâncias de delimitação, que são as regulamentações institucionais e as várias disciplinas que se envolvem no objeto em estudo; c) as análises de grades de especificação, que se referem aos sistemas que formam oposições, classificações e associações dos objetos e fenômenos em discurso.

\footnotetext{
${ }^{2} \mathrm{O}$ número entre parêntesis corresponde à quantidade de comunicações mapeadas por temáticas na pesquisa.
} 
Então, considera-se que a arqueologia foucaultiana se apresentou como um importante aporte procedimental na pesquisa sob o viés qualitativo, na medida em que possibilita maior amplitude metodológica em relação aos aspectos quantitativos obtidos por intermédio de análises bibliométricas.

Pretendeu-se identificar indicadores da produção colaborativa no domínio da arquitetura da informação através dos indícios que emergem pela Bibliometria. Para isto, descrevem-se analiticamente tais indicadores por meio de elementos paratextuais e textuais, a saber, data de publicação, GTs, autoria, instituições/programas de pós-graduação, e citações.

\section{CONDIÇões dA PRODUÇÃo COLABORATIVA NA ARQUITETURA DA INFORMAÇÃO}

As produções referentes à arquitetura da informação na Ciência da Informação se constituem por diversos aspectos, tais como a regularidade periódica em produções sobre a temática, vínculos por meio de GTs, compartilhamento de linguagens em comum e incomuns, pluralidade na formação acadêmica dos pesquisadores que abordam atemática, alguns indícios das convergências disciplinares a partir da colaboração em coautoria, bem como um plexo de relações através das citações indiretas que representam o compartilhamento de elementos teórico-metodológicos na produção interdisciplinar.

As comunicações sobre a arquitetura da informação no campo da Ciência da Informação se organizam de modo disperso por meio de vários termos indexadores, embora se perceba a concentração em um termo, no caso arquitetura da informação, conforme mostra a Tabela 1.

TABELA 1. Índice de comunicações por palavras-chave

\begin{tabular}{cc}
\hline Palavras-Chave & $f(x)$ \\
\hline Arquiteto da informação & 1 \\
Arquitetura da informação & 25 \\
Arquitetura de informação & 2 \\
Arquitetura da informação para web & 1 \\
Arquitetura da informação pervasiva & 1 \\
Arquitetura de informação - sistemas de organização do conhecimento & 1 \\
Arquitetura de metadados & 1 \\
\hline Total de Comunicações & $\mathbf{3 2}$ \\
\hline Fonte: dados da pesquisa (2015)
\end{tabular}

A partir disso, percebeu-se que as produções sobre esta temática se formam em dois movimentos distintos. A dispersão, visto que as comunicações se efetivam por meio de

\begin{tabular}{c|c|c|c|c|c}
\hline (C) RDBCI: Rev. Digit. Bibliotecon. Cienc. Inf. & Campinas, SP & v.15 & n.2 & p. 368-388 & maio/ago. 2017 \\
\hline
\end{tabular}


diferentes termos, e, por extensão, diversos significados devido à pluralidade na formação acadêmica dos pesquisadores da Ciência da Informação, sejam aqueles da Ciência da Computação, que se aproximam de subtemáticas como "metadados" ou "Web", ou da Biblioteconomia, que se relacionam aos "sistemas de organização do conhecimento".

Tratando os termos como indícios representativos dos conceitos, isto remete àquilo que Domingues (2005) chama de "traduzibilidade de conceitos". Isto acontece via operação de passagem de sentidos, uma vez que as palavras são utilizadas em diferentes campos daqueles originários (DOMINGUES, 2005). Assim, essa representatividade variada emerge em meio a deslocamentos de significados por intermédio de mudanças de termos em extensão e foco, de acordo com a abordagem da comunicação sobre a arquitetura da informação.

E na unidade, tendo em vista um efetivo compartilhamento de símbolos linguísticos em comum, na medida em que 25 (vinte e cinco) comunicações utilizam o mesmo termo arquitetura da informação. Isto refere àquilo que Japiassu (1976) pondera como "compartilhamento de linguagens em comum", aproximando-se, portanto, de uma das principais características da colaboração na produção do conhecimento científico.

Constata-se, conforme se observa na Tabela 2, que mais da metade do universo das comunicações sobre arquitetura da informação foi apresentada no GT8 Informação $e$ Tecnologia, que abarca estudos "teórico-práticos sobre e para o desenvolvimento de tecnologias de informação e comunicação que envolvam os processos de geração, representação, armazenamento, recuperação, disseminação, uso, gestão, segurança e preservação da informação em ambientes digitais" (ANCIB, 2014).

TABELA 2. Índice de comunicações por GT

\begin{tabular}{crr}
\hline GT & $\boldsymbol{f}(\mathbf{x})$ & $\boldsymbol{f}(\boldsymbol{\%})$ \\
\hline GT 1 & 1 & $3,13 \%$ \\
GT 2 & 2 & $6,25 \%$ \\
GT 3 & 2 & $6,25 \%$ \\
GT 4 & 2 & $6,25 \%$ \\
GT 6 & 2 & $6,25 \%$ \\
GT 8 & 20 & $62,50 \%$ \\
GT 9 & 1 & $3,13 \%$ \\
GT 11 & 2 & $6,25 \%$ \\
\hline Total & $\mathbf{3 2}$ & $\mathbf{1 0 0 , 0 0 \%}$ \\
\hline Fonte: dados da Pesquisa $(2015)$ &
\end{tabular}

Fonte: dados da Pesquisa (2015)

A arquitetura da informação é um domínio pragmático com enfoque na disseminação e representação de conteúdos em sistemas de informação para a Web. De acordo com autores como Agner (2009) e Morville e Rosenfeld (2006), esta temática possui uma base conceitual em torno dos processos de organização, representação, navegação e busca da informação, considerando as relações interdependentes entre conteúdos, contextos e usuários. 
No que tange à aproximação entre arquitetura da informação e tecnologia, entende-se que se trata de uma relação inerente, uma vez que esta temática decorre da avalanche tecnoinformacional e de discussões em relação à organização e à compreensão da informação em sistemas de informação, como ressalta Agner (2009).

Entretanto, tornam-se necessárias algumas revisões em torno dos conceitos e abordagens compreendidas nesta relação, na medida em que se possam trazer à tona questões sobre outras relações mais complexas como natureza-cultura, objeto-sujeito, intrinsecamente próximas às abordagens de arquiteturas informacionais. Nesse sentido, Santos e Vidotti (2009) ressaltam que a Ciência da Informação vem recebendo inúmeras críticas ao projeto que busca explicar e explicitar sua formação disciplinar a partir de dados, sistemas, algoritmos e símbolos destituídos de significados, isto é, aquelas bases teóricas que ponderam as tecnologias digitais simplesmente como ferramentas. Assim, as autoras afirmam que

a Ciência da Informação deveria ter ou criar mais espaços de investigação que permitam a compreensão das Tecnologias de Informação e Comunicação para a potencialização de competências informacionais, para a criação de arquiteturas informacionais e computacionais mais inclusivas, para a conceituação de usos da informação em ambientes informacionais digitais, para a aprendizagem de metalinguagens e para a representação da informação (SANTOS; VIDOTTI, 2009).

O domínio da arquitetura da informação na área requer discussões teóricas e práticas que considerem as tecnologias digitais como atores simbólicos de uma ação linguística. As tecnologias não são meros instrumentos, mas uma extensão daquilo que o homem constrói permeado por vários objetivos e sentidos. A informação, por exemplo, faz parte de uma ação múltipla entre vários dispositivos tecnológicos aplicados na linguagem, como comunicação simbólica e prática do homem na natureza.

Com efeito, há de se considerar os apontamentos de Saracevic (1999) dentro da Ciência da Informação, que afirma haver uma dificuldade na aproximação das vertentes focadas nos usuários/conteúdos e nas tecnologias por meio dos sistemas de recuperação da informação. Com base nisso, a arquitetura da informação emerge como domínio convergente dessas vertentes, visto que seus fundamentos teórico-metodológicos e as relações interdisciplinares, inicialmente em construção, possibilitam ponderar discussões em torno de específicos processos e fluxos de informação.

As comunicações do ENANCIB sobre a arquitetura da informação se estabelecem a partir de ações individuais e/ou coletivas, isto é, trata-se de produções realizadas individual ou coletivamente. A rigor, neste domínio analítico, a tipologia indicia a condição da produção colaborativa, quando esta é realizada em uma ação de coautoria.

Constatou-se um total de 59 (cinquenta e nove) autores que produzem sobre arquitetura da informação, no campo da Ciência da Informação. De acordo com indicadores bibliométricos

\begin{tabular}{c|c|c|c|c|c}
\hline (C) RDBCI: Rev. Digit. Bibliotecon. Cienc. Inf. & Campinas, SP & v.15 & n.2 & p. 368-388 & maio/ago. 2017 \\
\hline
\end{tabular}


apresentados no Gráfico 1, 27 (vinte e sete) comunicações foram realizadas em coautoria e apenas cinco constituíram-se em produções individuais. Os indicadores bibliométricos correspondentes à produção em coautoria (84\%) possibilitam perceber indícios do processamento efetivo da produção colaborativa na área.

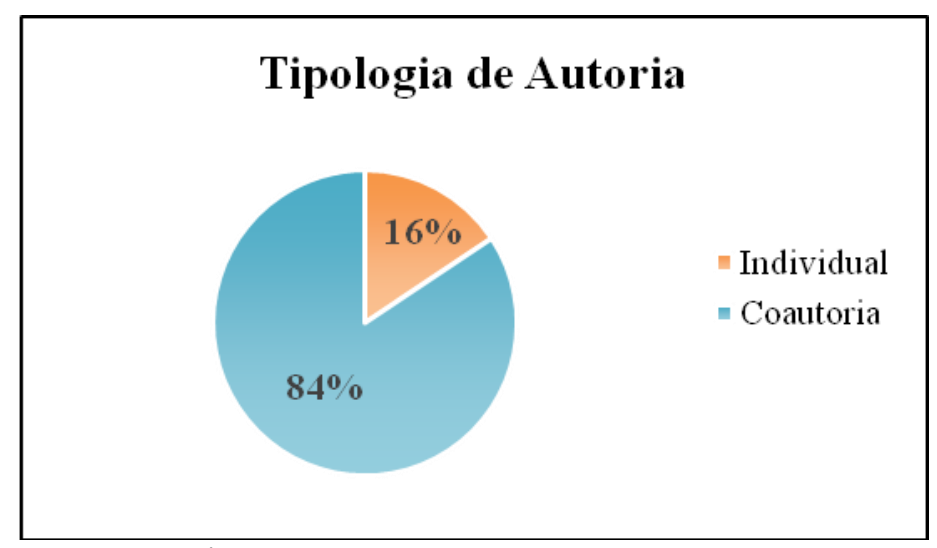

GRÁFICO 1. Índice de tipologia de autoria

Fonte: dados da pesquisa (2015)

Estas produções em coautoria se apresentam como condições para o estabelecimento de uma inteligência coletiva, que, para Domingues (2005), é uma ação entre indivíduos reunidos coletivamente.

Sendo assim, pôde-se afirmar, a princípio, que a produção científica na área sobre arquitetura da informação se estabelece a partir da união entre diferentes pesquisadores, portanto, como um processo multidisciplinar na colaboração científica. De acordo com Pombo (2008, p. 13), na multidisciplinaridade há

\footnotetext{
algum tipo de coordenação, numa perspectiva de mero paralelismo de pontos de vista. Algo que, quando se ultrapassa essa dimensão do paralelismo, do pôr em conjunto de forma coordenada, e se avança no sentido de uma combinação, de uma convergência, de uma complementaridade, nos coloca no terreno intermédio da interdisciplinaridade.
}

As comunicações que abordam a arquitetura da informação se concretizam por coordenação ou união de diferentes pesquisadores. Ainda assim, Domingues (2005) afirma que as justaposições não se efetivam necessariamente em produções interdisciplinares, mas formam condições efetivas de uma produção multidisciplinar, uma vez que se constituem por aproximações entre diferentes sujeitos (coautoria) e disciplinas coletivamente. Embora, observe-se a parceria existente entre orientador e orientando, consideram-se as relações que ultrapassam essa pareceria, buscando alcançar a dinâmica da colaboração teórico-metodológica a partir do compartilhamento de conceitos, teorias e métodos utilizados nas comunicações.

As produções colaborativas se efetivam em uma rede de relações conjuntamente entre coautoria e citações através de um jogo complexo de enunciados e frases, que, em menor ou maior grau, evidenciam compartilhamento de teorias e metodologias.

\begin{tabular}{c|c|c|c|c|c}
\hline (c) RDBCI: Rev. Digit. Bibliotecon. Cienc. Inf. & Campinas, SP & v.15 & n.2 & p. 368-388 & maio/ago. 2017 \\
\hline
\end{tabular}


A partir da Tabela 3, observou-se que as comunicações sobre arquitetura da informação se estabelecem em um plexo de relações de diferentes linguagens por meio de várias unidades de citações, totalizando 791 (setecentas e noventa e uma) citações das quais a maioria (64\%) é indireta.

TABELA 3. Índice de tipologia de citações

\begin{tabular}{cc}
\hline Tipologia & $\boldsymbol{f ( x )}$ \\
\hline Direta & 256 \\
Indireta & 502 \\
Citação de Citação & 33 \\
\hline Total & $\mathbf{7 9 1}$ \\
\hline
\end{tabular}

Fonte: dados da Pesquisa (2015)

Nessa categoria analítica, constatam-se as conexões com outros textos e as aproximações interpretativas de acordo com o contexto estabelecido. Assim, formam-se resultados referentes às interlinguagens por intermédio destas relações intertextuais. Vale lembrar as palavras de Japiassu (1976), ao afirmar que para a produção interdisciplinar tornase necessária em uma "interlinguagem" como um denominador comum, de modo que os pesquisadores se aproximem, buscando as relações fundamentais de contextos e conteúdos em torno das linguagens disciplinares em processo.

Entretanto, a partir de Etges (1995), entende-se que esta unidade intralinguística, a partir das relações de citações, não se configura somente por meio de relações específicas em linguagens comuns em menções extraídas de uma ou mais fontes iguais ou parecidas. É mais que isto, pois se configura a partir de dispersões e elementos aproximados intersubjetivamente, que se entrecruzam buscando ponderações críticas em torno de uma problemática e de um domínio específicos.

Ainda se constatou que os pesquisadores que comunicam artigos sobre arquitetura da informação na produção da Ciência da Informação estão vinculados a uma diversidade de áreas do conhecimento. Foi identificado um total de 27 (vinte e sete) disciplinas, considerando os três níveis acadêmicos, graduação, mestrado e doutorado.

A partir da pluralidade disciplinar presente na produção analisada, demonstrada no Gráfico 2, percebeu-se que duas áreas de conhecimento no nível de graduação ganham destaque - Biblioteconomia e Ciência da Computação. 


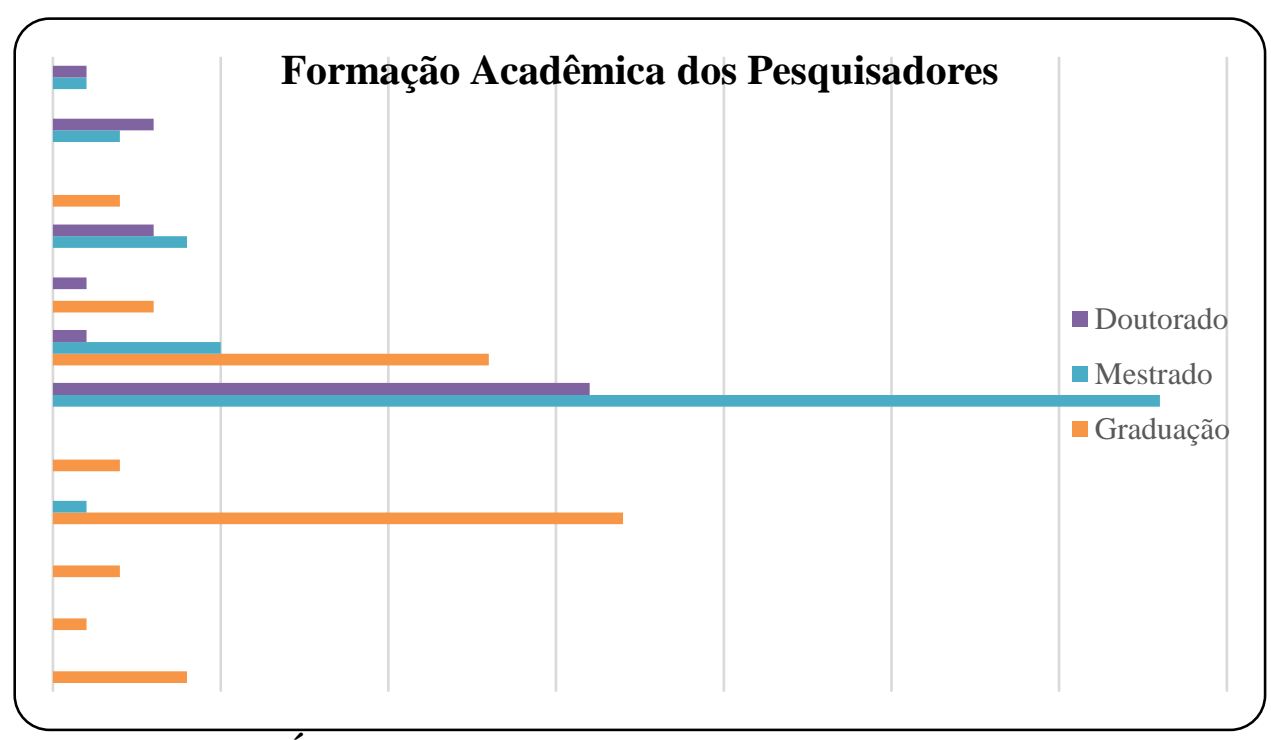

GRÁFICO 2. Formação Acadêmica dos Pesquisadores

Fonte: dados da pesquisa (2015)

Esse resultado se aproxima daquilo para que Saracevic $(1996,2009)$ chama a atenção, visto que a Biblioteconomia e a Ciência da Computação são áreas de conhecimento que fazem interface com a Ciência da Informação, e, mesmo assim, participam de dificuldades de convergência entre estas disciplinas devido às diferentes abordagens, respectivamente, centradas nos usuários e conteúdos e nos sistemas de recuperação da informação.

Neste ponto, compreende-se a necessidade de maiores aprofundamentos em relação às produções colaborativas sobre arquitetura da informação, tendo em vista as dificuldades de aproximações dessas duas áreas, apontadas por Saracevic (2009), visto que neste domínio emergem algumas discussões concernentes às abordagens consideradas dicotômicas, com enfoque no usuário (sujeito) e nos sistemas (objetos). A esse respeito, Santos e Vidotti (2009) propõem uma abordagem teórica focada no perspectivismo, na qual as tecnologias na Ciência da Informação não poderiam ser tratadas como ferramentais, a exemplo da Ciência da Computação, possibilitando, por intermédio da interdisciplinaridade e da transdisciplinaridade, diálogos transversais entre domínios diferentes atípicos de concepções dicotômicas entre sujeitos e objetos.

Para mais, esta pluralidade acadêmica, considerando as classificações de áreas, a partir da Coordenação de Aperfeiçoamento de Pessoal de Nível Superior - CAPES (2012), se constitui, sobretudo, em torno de duas grandes áreas, Ciências Exatas e da Terra e Ciências Sociais Aplicadas. Pode-se compreender que as justaposições que emergem em torno deste resultado são oriundas, principalmente, de duas áreas das quais fazem parte disciplinas como a Biblioteconomia, a Ciência da Computação e a Comunicação. Nesse sentido, para Saracevic (1996), estas disciplinas se aproximam da Ciência da Informação, tendo em vista, respectivamente, as técnicas de organização de registros bibliográficos, as peculiaridades do objeto informacional e as tecnologias da informação.

\begin{tabular}{c|c|c|c|c|c}
\hline (C) RDBCI: Rev. Digit. Bibliotecon. Cienc. Inf. & Campinas, SP & v.15 & n.2 & p. 368-388 & maio/ago. 2017 \\
\hline
\end{tabular}




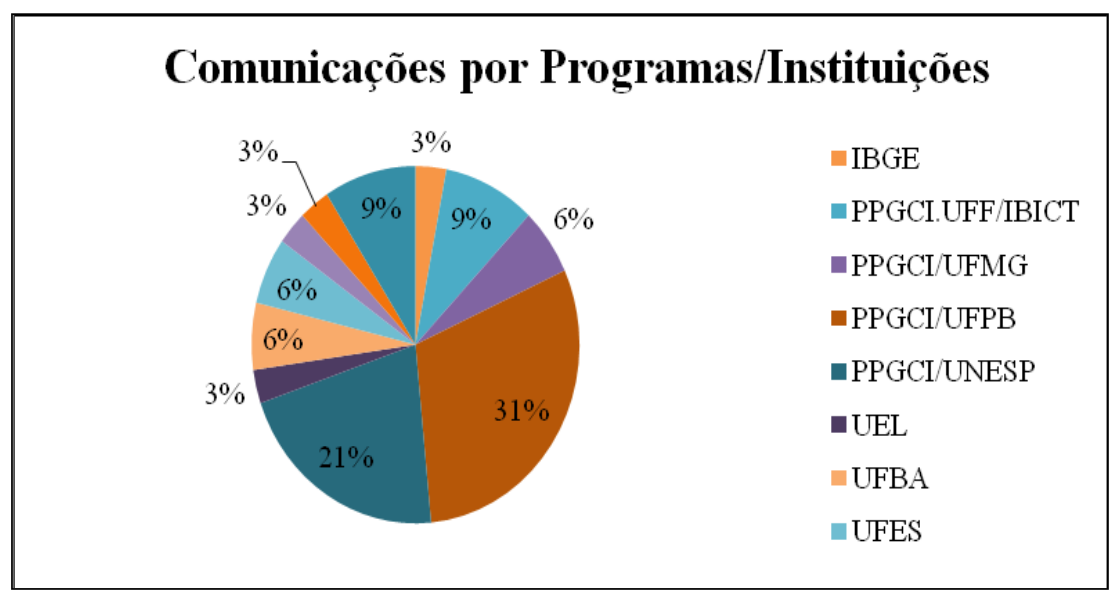

GRÁFICO 3. Índice de Comunicações por Programas/Instituições

Fonte: dados da pesquisa (2015)

Outro elemento importante diz respeito ao vínculo institucional das autorias. Assim, pode-se observar, no Gráfico 3, ainda, que as comunicações sobre arquitetura da informação no ENANCIB foram realizadas por autores de diferentes universidades e programas de pósgraduação em Ciência da Informação.

Ainda conforme Gráfico 3, contatou-se que os Programas de Pós-Graduação em Ciência da Informação (PPGCI) que apresentaram mais comunicações nos ENANCIBs, no período estudado, estão situados na Universidade Federal da Paraíba (UFPB) e na Universidade Estadual Paulista Júlio de Mesquita Filho (UNESP). Neste último, vale ressaltar que os autores Silvana Vidotti e Fernando Vechiato incidem diretamente no índice de produtividade de autores, pois representam o mesmo programa e contam com várias publicações em coautoria entre eles. $\mathrm{O}$ autor que mais publicou sobre a temática foi Marckson R. F. de Sousa da UFPB, como se vê na Tabela 4.

TABELA 4. Índice de produtividade de autores

\begin{tabular}{ccc}
\hline Autores & Programa/instituição & Comunicações $\boldsymbol{f}(\boldsymbol{x})$ \\
\hline André L. D. de França & PPGCI/UFPB & 2 \\
Fernando. L. Vechiato & PPGCI/UNESP & 3 \\
Guilherme. A. Dias & PPGCI/UFPB & 3 \\
Hellosman de O. Silva & PPGCI/UFPB & 2 \\
Lílian V. T. Cananéa & PPGCI/UFPB & 3 \\
Liriane S. A. Camargo & PPGCI/UNESP & 2 \\
Marckson R. F. de Sousa & PPGCI/UFPB & 7 \\
Maria A. T. da Silva & PPGCI/UFPB & 3 \\
Renata M.A. Baracho & PPGCI/UFMG & 2 \\
Silvana A. B. G. Vidotti & PPGCI/UNESP & 5 \\
\hline Total & - & $\mathbf{3 2}$ \\
\hline
\end{tabular}


Fonte: dados da pesquisa (2015)

É importante considerar que os programas da UFPB e UNESP contêm dentro de sua grade curricular de ensino no mínimo uma disciplina voltada ao domínio da arquitetura da informação, e consequentemente fazem parte destes programas os pesquisadores que mais publicam sobre esta temática, como pôde ser observado na Tabela 4.

Enfim, percebe-se que, de acordo com os indicadores bibliométricos, a produção científica do ENANCIB sobre a arquitetura da informação se estabelece em torno de vários elementos, corroborando para a emergência deste domínio no campo da Ciência da Informação, principalmente no que tange à produção colaborativa.

\section{CONSIDERAÇÕES FINAIS}

As comunicações realizadas no ENANCIB que tratam sobre a arquitetura da informação apresentam diferentes elementos que evidenciam as condições da produção colaborativa do conhecimento científico, que, apontam em maior ou menor medida, para a construção interdisciplinar do campo epistemológico da Ciência da Informação.

Considera-se que a subárea arquitetura da informação se configura como um domínio estratégico de produção colaborativa da Ciência da Informação, mesmo que em um espaço conflituoso e epistêmico, tendo em vista a dificuldade de integração entre duas vertentes na Ciência da Informação, apontadas por Saracevic (1999). Trata-se de considerar as condições de produção, que compreendem a tipologia de autor, a pluralidade de formação acadêmica, o compartilhamento de linguagens e as aproximações institucionais, que caracterizam a produção colaborativa sobre a arquitetura da informação.

Os indicadores bibliométricos permitiram observar indícios da produção interdisciplinar na arquitetura da informação. As comunicações sobre esta temática se efetivam por meio de vários autores, portanto, como produções colaborativas, e, por conseguinte, através de uma pluralidade acadêmica, tendo em vista as disciplinas representadas pelos pesquisadores envolvidos. Assim, por intermédio de justaposições entre diferentes disciplinas e áreas do conhecimento científico que compreendem processos multidisciplinares no campo da Ciência da Informação, especificamente no domínio da arquitetura da informação.

Além disso, se estendem em indícios de convergência disciplinar a partir da integração de símbolos linguísticos, como os termos de indexação e as citações indiretas, podendo se efetivar no compartilhamento de elementos teórico-metodológicos, que fundamentam a produção interdisciplinar na área.

A rigor, por meio dos indicadores bibliométricos, foi possível descrever, conforme Foucault (2008), algumas superfícies de emergência e instâncias de delimitação da produção estudada. As primeiras objetivaram a descrição do domínio analítico na arquitetura da 
informação, dando-lhe condições básicas de um objeto do campo da Ciência da Informação, e compreenderam elementos paratextuais e textuais (GTs, autores, termos de indexação e citações). As segundas, por sua vez, buscaram identificar as regulamentações institucionais e disciplinares que se envolvem da produção colaborativa da arquitetura da informação, na área da Ciência da Informação. Estas compreenderam as disciplinas, as áreas do conhecimento, as universidades e os programas de pós-graduação à que se refere a produção estudada.

De modo geral, os resultados evidenciam os elementos e suas respectivas características que condicionam a produção colaborativa na área, especificamente em comunicações nos domínios da arquitetura da informação. Entretanto, entende-se que, em relação às discussões sobre a produção interdisciplinar na Ciência da Informação, há algumas dificuldades e podem ser ampliadas no que tange aos procedimentos teórico-metodológicos que possibilitem empiricamente reflexões mais claras sobre a processualidade e a efetividade das produções colaborativas, podendo observar não só as condições em que se processam estas atividades, mas, sobretudo, as bases constituintes que fundamentam a interdisciplinaridade no conhecimento científico. Afinal, como afirma Japiassu (1976), a interdisciplinaridade se configura a partir da integração de conceitos, teorias, metodologias e linguagens entre diferentes disciplinas.

Vale ressaltar, também, que, analisando as condições da produção interdisciplinar na Ciência da Informação tendo em vista suas características, a complexidade da informação, a pluralidade acadêmica e a consequente convergência disciplinas, percebe-se que os estudos sobre a interdisciplinaridade na área podem tentar compreender os movimentos de unidade e dispersão. Pois as discussões sobre o compartilhamento de elementos teórico-metodológicos não se findam em fechar dogmaticamente ou abrir a qualquer custo as relações interdisciplinares, mas, sobretudo, ponderar as bases constitutivas da produção interdisciplinar na Ciência da Informação, que contemplam, especificamente, os fundamentos e as estratégias, ou, de modo mais preciso, os conceitos, as teorias, as metodologias e as linguagens, entre outros.

Em última análise, considera-se que a produção colaborativa na arquitetura da informação emerge como um espaço epistêmico estratégico da produção interdisciplinar no campo da Ciência da Informação, possibilitando maiores reflexões sobre a convergência disciplinar entre diferentes disciplinas e áreas do conhecimento, considerando a complexidade do objeto informacional e os processos e fluxos informacionais que o envolvem.

\section{REFERÊNCIAS}

AGNER, Luiz. Ergodesign e arquitetura de informação: trabalhando com o usuário. 2. ed. Rio de Janeiro: Quartet, 2009.

ALVARENGA, Lídia. Bibliometria e arqueologia do saber de Michel Foucault: traços de identidade teórico-metodológica. Ciência da Informação, v. 27, n. 3, set. 1998. Disponível 
em: 〈http://www.scielo.br/scielo.php?pid=S0100-19651998000300002\&script=sci_arttext $>$. Acesso em: 11 maio. 2015.

\section{ASSOCIAÇÃO NACIONAL DE PESQUISA E PÓS-GRADUAÇÃO M CIÊNCIA DA} INFORMAÇÃO. GT 08 - Informação e Tecnologia. Ementa. Disponível em: <http://gtancib.fci.unb.br/index.php/gt-08 >. Acesso em: 20 out. 2014.

BRASIL. Ministério da Educação. Coordenação de Aperfeiçoamento de Pessoal de Nível Superior. Tabela de áreas de conhecimento/avaliação. Brasília: Capes, 2012. Disponível em: 〈http://www.capes.gov.br/avaliacao/instrumentos-de-apoio/tabela-de-areas-doconhecimento-avaliacao >. Acesso em: 11 abr. 2015.

CAPURRO, Rafael.; HJØRLAND, Birger. O conceito de informação. Perspectivas em Ciência da Informação, Belo Horizonte, v. 12, n. 1, p. 148-207, jan. /abr., 2007. Disponível em: 〈http://www.scielo.br/pdf/pci/v12n1/11.pdf $>$. Acesso em: 8 abr. 2015.

DOMINGUES, Ivan. Conhecimento e transdisciplinaridade II: aspectos metodológicos. Belo Horizonte: Editora UFMG, 2005.

ETGES, Norberto Jacob. Ciência, interdisciplinaridade e educação. In: JANTSCH, Ari Paulo.; BIANCHETTI, Lucídio. (Org.). Interdisciplinaridade: para além da filosofia do sujeito. Petrópolis, RJ: Vozes, 1995. p. 51-84., cap. 3.

FOUCAULT, Michel. A arqueologia do saber. 7. ed. Rio de Janeiro: Forense Universitária, 2008.

GONZÁLEZ DE GÓMEZ, Maria Nélida. Para uma reflexão epistemológica acerca da Ciência da Informação. Perspectivas em Ciência da Informação, Belo Horizonte, v. 6, n. 1, p. 5-18, jan./jun. 2001. Disponível em: <http://portaldeperiodicos.eci.ufmg.br/index.php /pci/article/view/433>. Acesso em: 15 jul. 2014.

JAPIASSU, Hilton. Interdisciplinaridade e patologia do saber. Rio de Janeiro: Imago, 1976.

LARA, Marilda Lopes Ginez de.; SMIT, Johanna Wilhelmina. Os Enancibs e a Ciência da Informação brasileira: introdução. In: LARA, Marilda Lopes Ginez de.; SMIT, Johanna Wilhelmina. (Org.). Temas de pesquisa em Ciência da Informação no Brasil. São Paulo: USP, 2010.

MACEDO, Flávia Lacerda Oliveira. Arquitetura da informação: aspectos epistemológicos, científicos e práticos. 2005.187 f. Dissertação (Mestrado em Ciência da Informação) Universidade de Brasília, Brasília, 2005.

\begin{tabular}{c|c|c|c|c|c}
\hline (c) RDBCI: Rev. Digit. Bibliotecon. Cienc. Inf. & Campinas, SP & v.15 & n.2 & p. 368-388 & maio/ago. 2017 \\
\hline
\end{tabular}


MACÍAS-CHAPULA, Cesar Augusto. O papel da informetria e da cientometria e sua perspectiva nacional e internacional. Ciência da Informação, Brasília, v. 27, n. 2, p. 134140, maio/ago. 1998. Disponível em: 〈http://goo.gl/H09oKH>. Acesso em: 10 jul. 2015.

MORVILLE, Peter.; ROSENFELD, Louis. Information architecture for the World Wide Web. 3.ed. Sebastopol: O’Really, 2006.

PINHEIRO, Lena Vania Ribeiro. A ciência da informação entre a sombra e a luz: domínio epistemológico e campo interdisciplinar. 1997. Tese (Doutorado em Ciência da Informação) - Escola de Comunicação, Universidade Federal do Rio de Janeiro, Rio de Janeiro, 1997.

POMBO, Olga. Epistemologia da interdisciplinaridade. Ideação - Revista do Centro de Educação e Letras da UNIOESTE, Foz do Iguaçu, v. 10, n. 1, p. 9-40, 2008.

SANTOS, Plácida Leopoldina Ventura Amorim da Costa.; VIDOTTI, Silvana Aparecida Borsetti Gregorio. Perspectivismo e tecnologias de informação e comunicação: acréscimos à Ciência da Informação? Datagramazero - Revista de Ciência da Informação, Rio de Janeiro, v. 10, n. 3, p. 1-10, jun. 2009. Disponível em:

<http://www.dgz.org.br/jun09/Art_02.htm>. Acesso em: 15 abr. 2015.

SARACEVIC, Tefko. Ciência da Informação: origens, evolução e relações. Perspectivas em Ciência da Informação, Belo Horizonte, v. 1, n. 1, p. 41-62, jan./jun. 1996. Disponível em: <http://portaldeperiodicos.eci.ufmg.br/index.php/pci/article/view/235/22 >. Acesso em: 11 abr. 2014.

SARACEVIC, Tefko. Information Science. Journal of the American Society for Information Science, New. York, v. 50, n. 12, p. 1051-1063, 1999. Disponível em: <https://comminfo.rutgers.edu/ tefko/JASIS1999.pdf>. Acesso em: 21 abr. 2015.

SOUZA, Edivanio Duarte de. A epistemologia interdisciplinar na Ciência da Informação: dos indícios aos efeitos de sentido na consolidação do campo disciplinar. 2011. $346 \mathrm{f}$. Tese (Doutorado em Ciência da Informação) - Escola de Ciência da Informação, Universidade Federal de Minas Gerais, Minas Gerais, 2011.

SOUZA, Edivanio Duarte de. A epistemologia interdisciplinar: uma introdução à produção colaborativa de conhecimento científico. Maceió: Edufal, 2015.

WURMAN, Richard. Information architects. 2. ed. Lakewood: Watson-Guptill Pubns, 1997. 


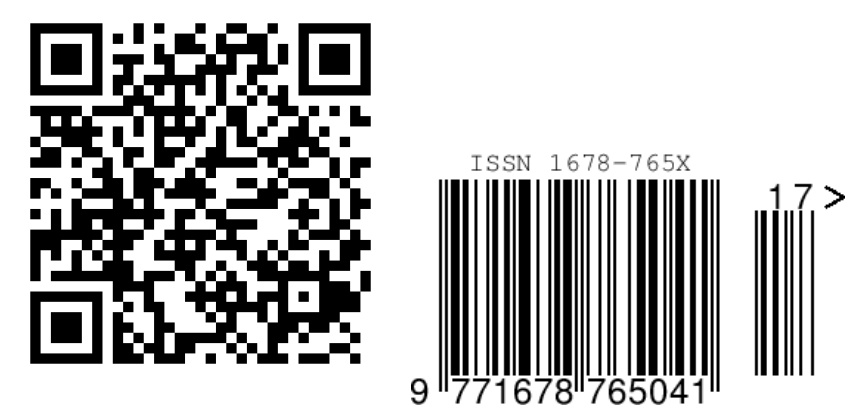

